\title{
Simultaneous extraction of geometry and surface properties of targets using simple infrared sensors
}

\author{
Tayfun Aytaç \\ Billur Barshan \\ Bilkent University \\ Department of Electrical Engineering \\ TR-06800 Bilkent, Ankara, Turkey \\ E-mail: billur@ee.bilkent.edu.tr
}

\begin{abstract}
We investigate the use of low-cost infrared (IR) sensors for the simultaneous extraction of geometry and surface properties of commonly encountered features or targets in indoor environments, such as planes, corners, and edges. The intensity measurements obtained from such sensors are highly dependent on the location, geometry, and surface properties of the reflecting target in a way that cannot be represented by a simple analytical relationship, therefore complicating the localization and recognition process. We propose the use of angular intensity scans and present an algorithm to process them to determine the geometry and the surface type of the target and estimate its position. The method is verified experimentally with planes, 90-deg corners, and 90-deg edges covered with aluminum, white cloth, and Styrofoam packaging material. An average correct classification rate of $80 \%$ of both geometry and surface over all target types is achieved and targets are localized within absolute range and azimuth errors of $1.5 \mathrm{~cm}$ and 1.1 deg, respectively. Taken separately, the geometry and surface type of targets can be correctly classified with rates of 99 and $81 \%$, respectively, which shows that the geometrical properties of the targets are more distinctive than their surface properties, and surface determination is the limiting factor. The method demonstrated shows that simple IR sensors, when coupled with appropriate processing, can be used to extract substantially more information than that for which such devices are commonly employed. (c) 2004 Society of Photo-Optical Instrumentation Engineers. [DOI: 10.1117/1.1789136]
\end{abstract}

Subject terms: pattern recognition; feature extraction; target differentiation; target localization; surface differentiation; surface localization; infrared sensors; position estimation; optical sensing.

Paper 030547 received Oct. 31, 2003; revised manuscript received Mar. 11, 2004; accepted for publication Mar. 28, 2004.

\section{Introduction}

Target differentiation and localization is of considerable interest for intelligent systems where it is necessary to identify targets and their positions for autonomous operation. Differentiation is also important in industrial applications where different materials must be identified and separated. In this paper, we consider the use of a very simple IR sensing system consisting of one emitter and one detector for the purpose of differentiation and localization. These devices are inexpensive, practical, and widely available. The emitted light is reflected from the target and its intensity is measured at the detector. However, it is often not possible to make reliable distance estimates based on the value of a single intensity return because the return depends on both the geometry and surface properties of the reflecting target. Likewise, the properties of the target cannot be deduced from simple intensity returns without knowing its distance and angular location. In this paper, we propose a scanning technique and an algorithm that can simultaneously determine the geometry and the surface type of the target, in a manner that is invariant to its location. Once the target type is determined, its position $(r, \theta)$ can also be estimated. The method we propose is scalable in the sense that the accuracy can be increased by increasing the number of reference scans without increasing the computational complexity of the differentiation and localization process. Our results show that by properly processing data obtained from such simple IR sensors, it is possible to extract a significantly greater amount of information than is commonly expected from such sensors.

Most work on pattern recognition involving IR deals with recognition or detection of features or targets in conventional 2-D images. Examples of work in this category include face identification, ${ }^{1}$ automatic target recognition, ${ }^{2}$ target tracking, ${ }^{3}$ automatic vehicle detection, ${ }^{4}$ remote sensing, ${ }^{5}$ detection and identification of targets in background clutter, ${ }^{6,7}$ and automated terrain analysis. ${ }^{8}$ Note that the position-invariant pattern recognition and position estimation achieved in this paper are different from such operations performed on conventional images ${ }^{9}$ in that here we work not on direct "photographic" images of the targets obtained by some kind of imaging system, but rather on angular intensity scans obtained by rotating a point sensor. The targets we differentiate are not patterns in a 2-D image 
whose coordinates we try to determine, but rather objects in space, exhibiting depth, whose position with respect to the sensing system we must estimate. As such, positioninvariant differentiation and localization is achieved with an approach quite different than those employed in invariant pattern recognition and localization in conventional images. ${ }^{10-16}$

IR sensors are used in robotics and automation, process control, remote sensing, and safety and security systems. More specifically, they have been used in simple object and proximity detection, ${ }^{17}$ counting, ${ }^{18}$ distance and depth monitoring, ${ }^{19}$ floor sensing, position measurement, and control, ${ }^{20,21}$ obstacle/collision avoidance, ${ }^{22,23}$ and map building. ${ }^{24}$ IR sensors are used in door detection and mapping of openings in walls, ${ }^{25}$ as well as monitoring doors/ windows of buildings and vehicles, and "light curtains" for protecting an area. In Ref. 26, the properties of a planar surface at a known distance were determined using the Phong illumination model, and using this information, the IR sensor employed was modeled as an accurate range finder for surfaces at short ranges. In Ref. 27, an IR-sensorbased system that can measure distances up to $1 \mathrm{~m}$ is described. References 28, 29, and 30 deal with optical determination of depth information. In Ref. 31, simulation and evaluation of the recognition abilities of active IR sensor arrays are considered for autonomous systems using a raytracing approach. Reference 32 describes a passive IR sensing system that identifies the locations of the people in a room. IR sensors have also been used for automated sorting of waste objects made of different materials. ${ }^{33}$ In Ref. 34, we considered targets with different geometrical properties but made of the same surface material (wood). A correct classification rate of $97 \%$ was achieved with absolute range and azimuth errors of $0.8 \mathrm{~cm}$ and $1.6 \mathrm{deg}$. A rule-based approach to the same problem can be found in Ref. 35. In Ref. 36, targets made of different surface materials but of the same planar geometry were differentiated with a correct differentiation rate of $87 \%$ and absolute range and azimuth errors of $1.2 \mathrm{~cm}$ and $1.0 \mathrm{deg}$. In this paper, we deal with the problem of differentiating and localizing targets whose geometry and surface properties both vary, generalizing and unifying the results of Refs. 34 and 36.

\section{Target Differentiation and Localization}

The IR sensor ${ }^{37}$ used in this study [see Fig. 1(a)] consists of an emitter and detector, works with 20 to $28-\mathrm{V}$ dc input voltage, and provides analog output voltage proportional to the measured intensity reflected off the target. The detector window is covered with an IR filter to minimize the effect of ambient light on the intensity measurements. Indeed, when the emitter is turned off, the detector reading is essentially zero. The sensitivity of the device can be adjusted with a potentiometer to set the operating range of the system.

The targets employed in this study are a plane, a 90-deg corner, and a 90-deg edge, each with a height of $120 \mathrm{~cm}$ (Fig. 2). They are covered with aluminum, white cloth, and Styrofoam packaging material. Our method is based on angularly scanning each target over a certain angular range. The IR sensor is mounted on a 12 -in. rotary table ${ }^{38}$ to obtain angular scans from these targets. A photograph of the experimental setup and its schematics can be seen in Figs.

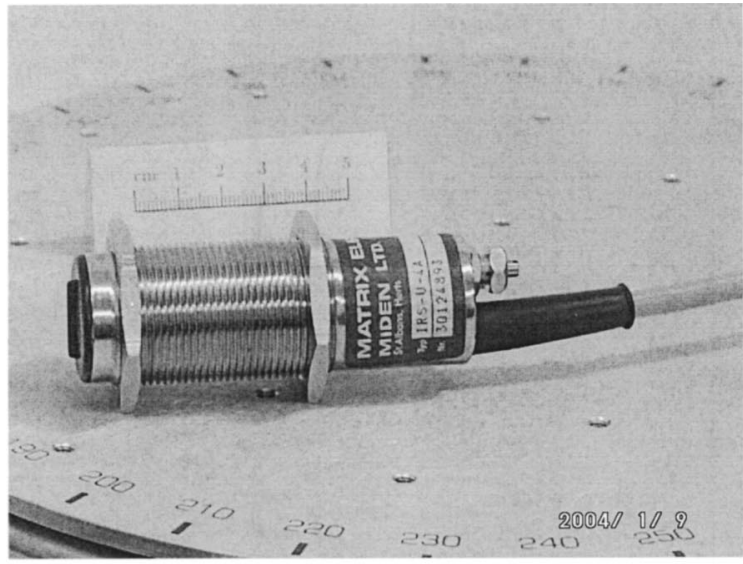

(a)

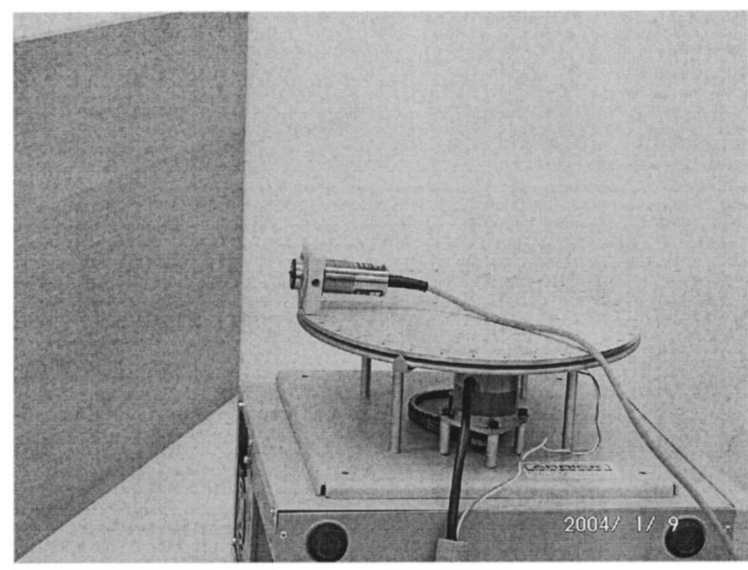

(b)

Fig. 1 (a) IR sensor used in this study and (b) experimental setup.

1(b) and 3, respectively. Reference data sets are collected for each target with $2.5-\mathrm{cm}$ distance increments from their nearest to their maximum observable ranges at $\theta=0 \mathrm{deg}$. The output signal is processed using an 8-bit microprocessor-compatible analog-to-digital converter chip having a conversion time of $100 \mu \mathrm{s}$.

The resulting reference scans for the plane, the corner, and the edge covered with materials of different surface properties are shown in Figs. 4, 5, and 6. The intensity scans are $\theta$ invariant but not $r$ invariant; changes in $r$ result in variations in both the magnitude and the basewidth of the intensity scans. Scans of corners covered with white cloth and Styrofoam packaging material have a triple-humped

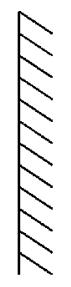

plane

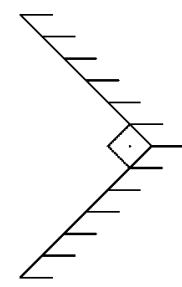

corner

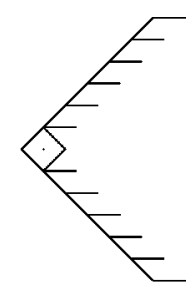

edge
Fig. 2 Target primitives used in this study. 
Aytaç and Barshan: Simultaneous extraction of geometry ...

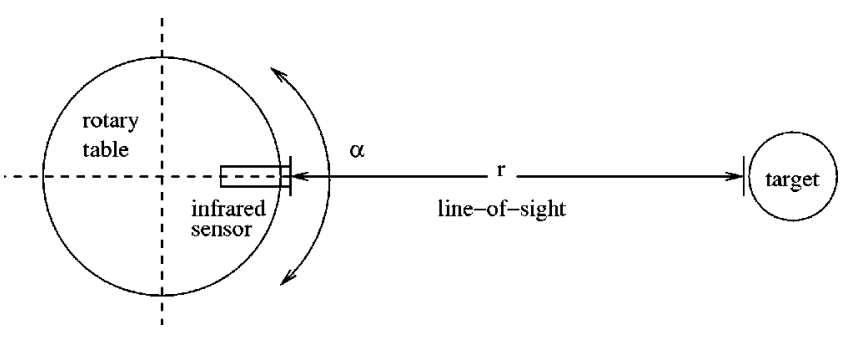

Fig. 3 Top view of the experimental setup used in target differentiation and localization. The emitter and detector windows are circular with an 8-mm diameter and a center-to-center separation $12 \mathrm{~mm}$. (The emitter is above the detector.) Both the scan angle $\alpha$ and the target azimuth $\theta$ are measured counterclockwise from the horizontal axis.

pattern (with a much smaller middle hump) corresponding to the two orthogonal constituent planes and their intersection. The intensity scans for corners covered with aluminum [Fig. 5(a)] have three distinct saturated humps. Notice that the return signal intensities saturate at an intensity corresponding to about $11 \mathrm{~V}$ output voltage.

We now describe the differentiation and localization process of an arbitrarily located target whose intensity scan was observed. First, we check for saturation by examining

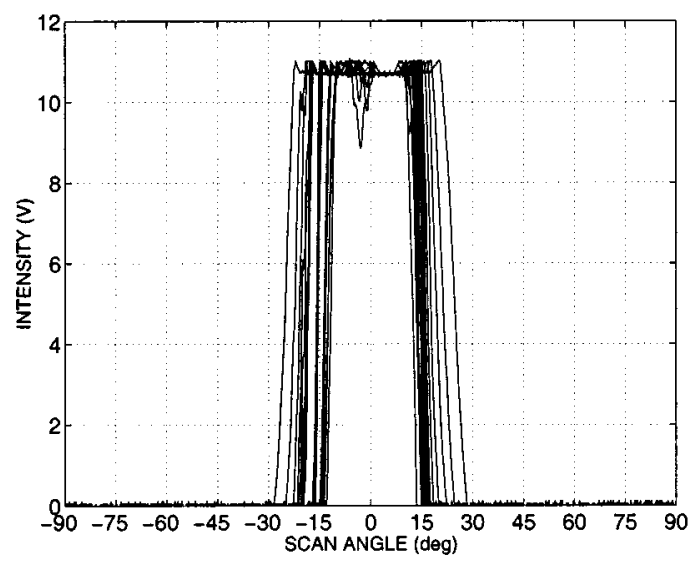

(a) the central intensity value of the observed scan $I(\alpha)$. This situation is treated separately, as will be explained later in Sec. 2.3. Note that a corner scan is considered saturated when its central intensity enters the saturation region, not the humps, since it is the former value that is relevant for our method.

We start by determining the target type. Unfortunately, direct comparison with the corresponding curves in Figs. 4-6 is not possible since we do not yet know the distance to the target, and comparing with all the curves at all distances would be computationally very expensive. Therefore, we exploit the fact that the successive curves in Figs. 4-6 exhibit a monotonic dependence on distance. Furthermore, when an observed scan is compared to the several successive curves in any of Figs. 4-6, the two measures of difference between them described in Secs. 2.1 and 2.2 also exhibit a monotonic fall and rise around a single minimum. Therefore, we are ensured that we will not be settling at a suboptimal point if we compare the observed scan not with all scans at all distances, but only with the nine scans (one for each particular geometry and surface type) whose central intensities are closest to that of the observed scan. Therefore, for unsaturated scans, it is sufficient to make nine comparisons instead of comparisons with all the scans

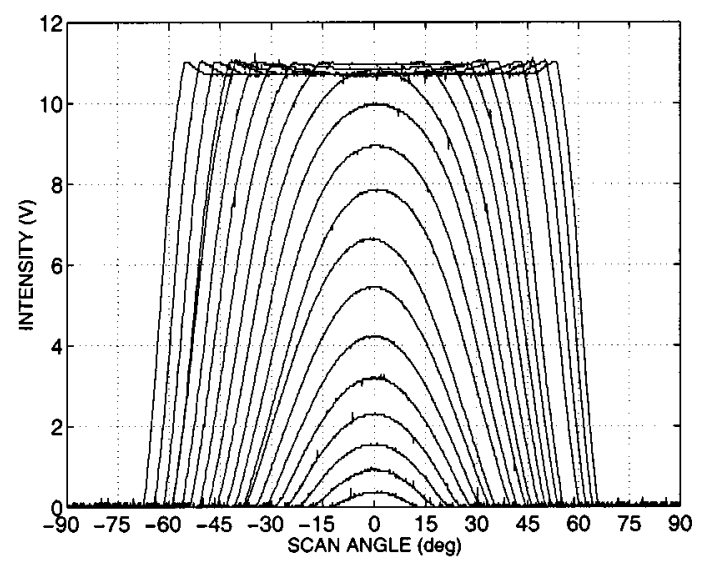

(b)

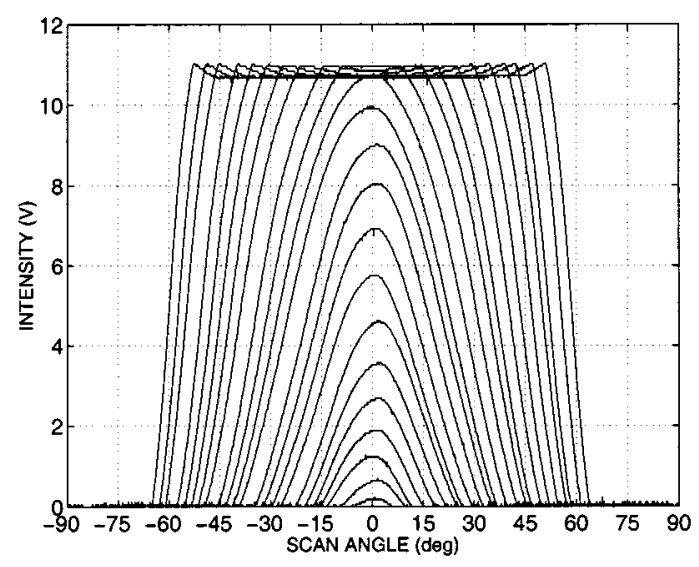

(c)

Fig. 4 Intensity scans for planes at different distances covered with different surface materials: (a) aluminum, (b) white cloth, and (c) Styrofoam. 
Aytaç and Barshan: Simultaneous extraction of geometry ...

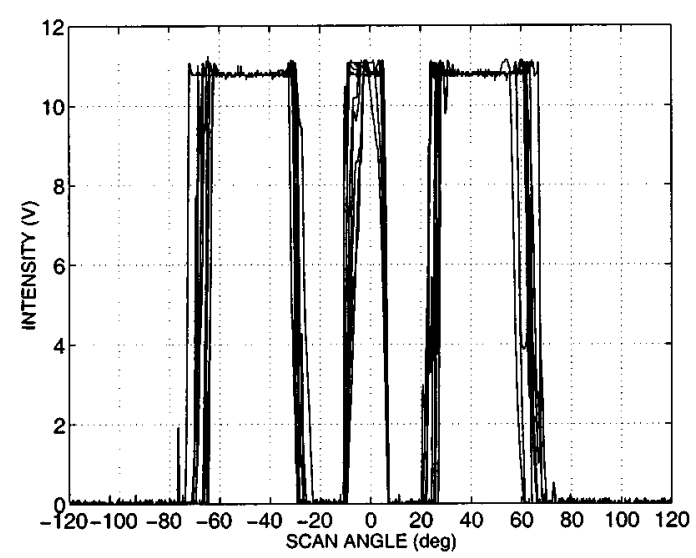

(a)

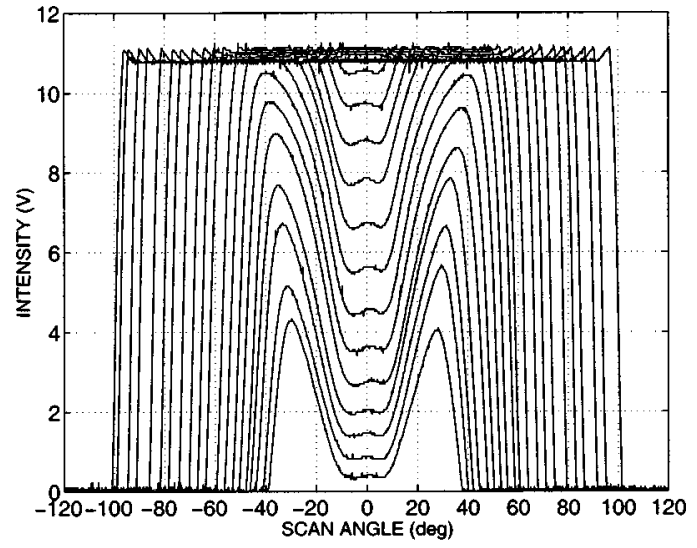

(b)

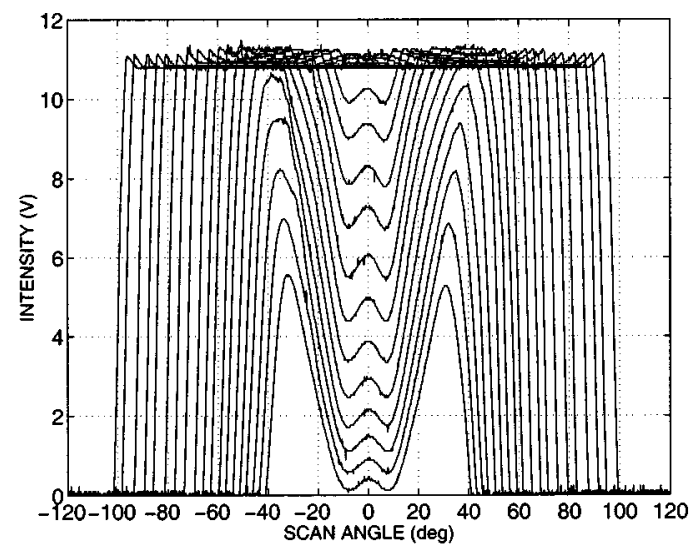

(c)

Fig. 5 Intensity scans for corners at different distances covered with different surface materials: (a) aluminum, (b) white cloth, and (c) Styrofoam.

in Figs. 4-6. This remains the case even if the 2.5-cm increments are reduced to smaller values. This has the advantage that the accuracy of the system can be increased without increasing the cost of computation (although a greater number of scans must be stored). As a test, we also ran a version of the method where 18 comparisons were made using the scans with the nearest central intensities both above and below the observed central intensity, and also using all of the scans shown in Figs. 4-6. These computationally more expensive approaches, exceedingly more so in the latter case, did not improve the results with respect to a comparison with only nine scans. In fact, in the matched filtering case discussed in Sec. 2.2, the results are even somewhat better when nine scans are used, due to the fact that this systematic elimination of a priori suboptimal scans eliminates the small possibility that they will mistakenly be chosen as the best matching scan due to noise and other errors.

Two alternative approaches are employed in performing the nine comparisons. These are discussed in the following two subsections.

\subsection{Least-Squares Approach}

First, we estimate the angular position of the target as follows. Assuming the observed scan pattern is not saturated, we check whether or not it has two major humps. If so, it is a corner and we find the angular location of the corner by taking the average of the angular locations of the peaks of the two major humps of the intensity scan. If not, we find the angular location of the peak of the single hump. This angular value can be directly taken as an estimate of the angular position of the target. Alternatively, the angular position can be estimated by finding the center of gravity (COG) of the scan as follows:

$\theta_{\mathrm{COG}}=\frac{\sum_{i=1}^{n} \alpha_{i} I\left(\alpha_{i}\right)}{\sum_{i=1}^{n} I\left(\alpha_{i}\right)}$.

Ideally, these two angular position estimates would be equal, but in practice they differ by a small amount. We consider the use of both alternatives when tabulating our results. From now on, we refer to either estimate as the "center angle" of the scan.

Plots of the intensity at the center angle of each scan in Figs. 4-6 as a function of the distance at which that scan was obtained, play an important role in our method. Figure 7 shows these plots for the intensity value at the COG for planes, corners, and edges. 
Aytaç and Barshan: Simultaneous extraction of geometry ...

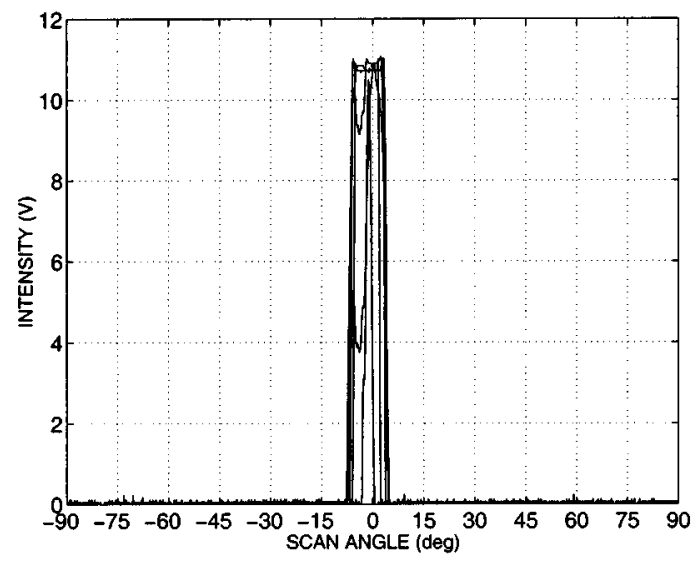

(a)

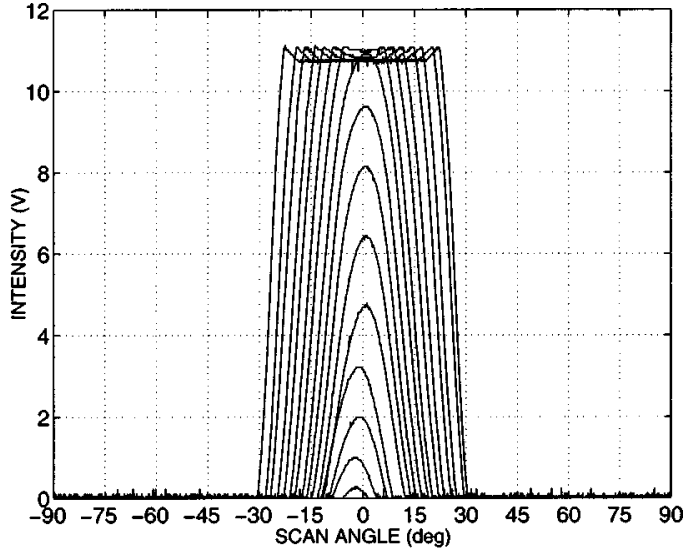

(b)

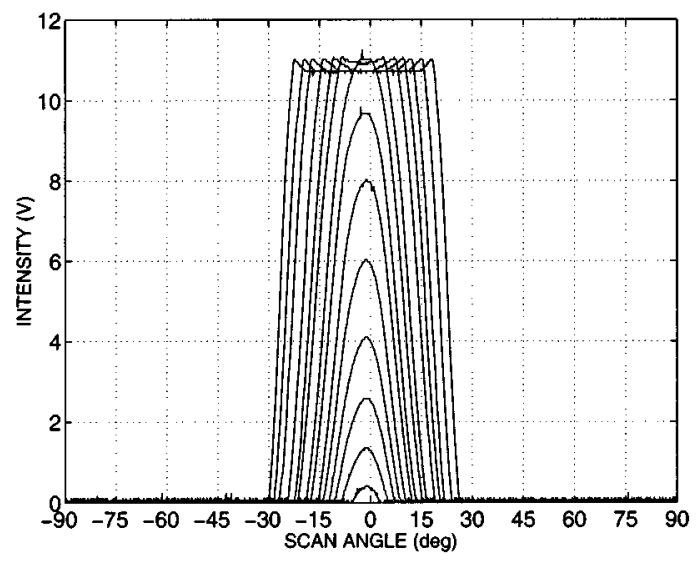

(c)

Fig. 6 Intensity scans for edges at different distances covered with different surface materials: (a) aluminum, (b) white cloth, and (c) Styrofoam.

In this approach, we compare the intensity scan of the observed target with the nine reference scans by computing their least-squares differences after aligning their centers with each other. The mean-square difference between the observed scan and the nine scans is computed as follows:

$\mathcal{E}_{j}=\frac{1}{n} \sum_{i=1}^{n}\left[I\left(\alpha_{i}-\alpha_{\text {align }}\right)-I_{j}\left(\alpha_{i}\right)\right]^{2}$,

where $I_{j}, j=1, \ldots, 9$, denotes the nine scans. Here, $\alpha_{\text {align }}$ is the angular shift that is necessary to align both patterns. The geometry-surface combination resulting in the smallest value of $\mathcal{E}$ is declared as the observed target. Once the geometry and surface type are determined, the range can be estimated by using linear interpolation on the appropriate curve in Fig. 7. Note that, in this way, the accuracy of the method is not limited by the $2.5-\mathrm{cm}$ spacing used in collecting the reference scans.

\subsection{Matched Filtering Approach}

As an alternative, we also considered the use of matched filtering ${ }^{39}$ to compare the observed and reference scans. The output of the matched filter is the cross-correlation between the observed intensity pattern and the $j$ 'th reference scan normalized by the square root of its total energy:

$y_{j}(l)=\frac{\sum_{k} I\left(\alpha_{k}\right) I_{j}\left(\alpha_{k-l}\right)}{\left\{\sum_{i=1}^{n}\left[I_{j}\left(\alpha_{i}\right)\right]^{2}\right\}^{1 / 2}}$,

where $l=1, \ldots, 2 n-1$ and $j=1, \ldots, 9$. The geometry-surface combination corresponding to the maximum crosscorrelation peak is declared as the correct target type, and the angular position of the correlation peak directly provides an estimate of the azimuth angle of the target. Then, the distance is estimated by using linear interpolation on the appropriate curve in Fig. 7 using the intensity value at the azimuth estimate.

\subsection{Saturated Scans}

If saturation is detected in the observed scan, special treatment is necessary. In the least-squares approach, the mean square differences between the aligned observed scan and all the saturated reference scans are computed and the target type with the minimum mean square difference is chosen. The range estimate of the target is taken as the distance corresponding to the scan resulting in the minimum mean 


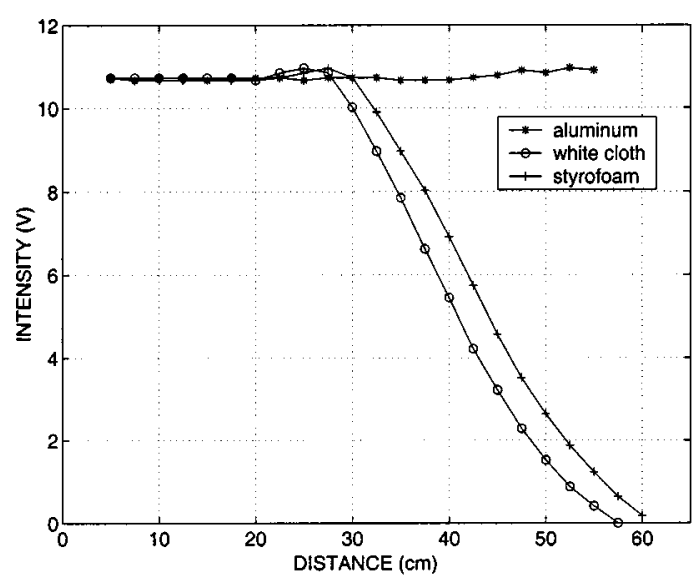

(a)

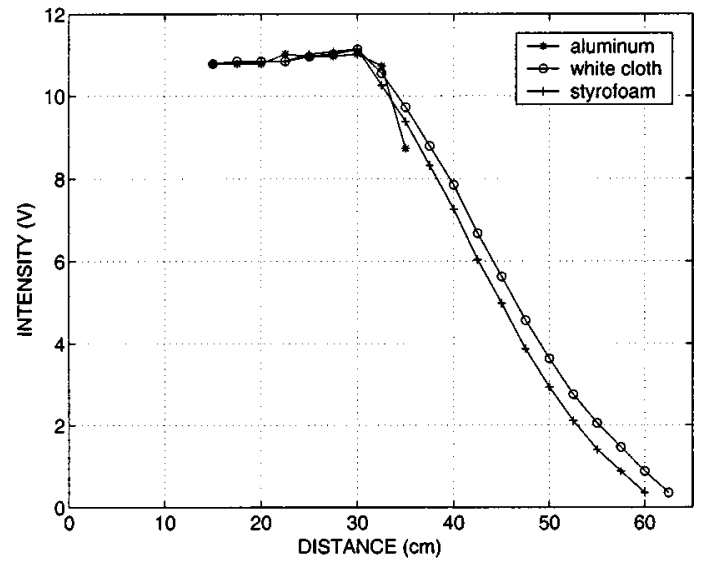

(b)

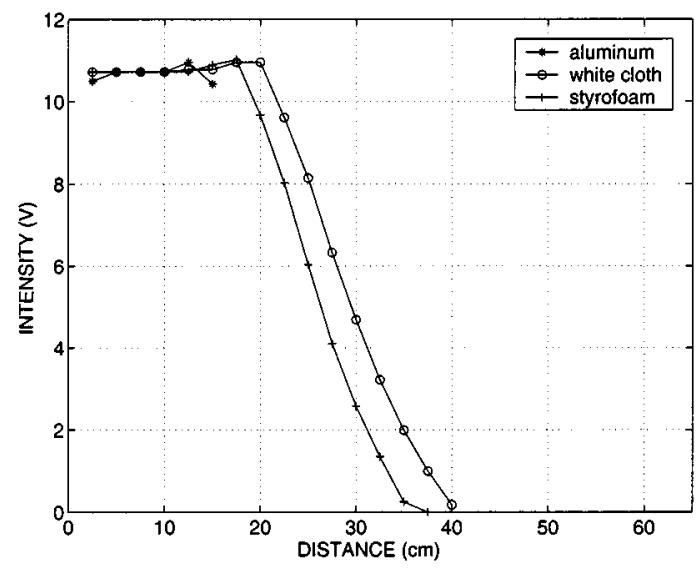

(c)

Fig. 7 Central intensity (COG) versus distance curves for different targets: (a) plane, (b) corner, and (c) edge.

square difference. Similarly, for the matched filter, correlation between the observed scan and all the stored saturated reference scans is computed and the target type resulting in the highest correlation peak is selected. The range estimate is again taken as that of the best matching scan.

Note that, in the saturated case, range estimation accuracy is limited by the $2.5-\mathrm{cm}$ interval at which the reference scans were taken since interpolation is not possible. If the accuracy is not satisfactory, it can be improved by reducing the $2.5-\mathrm{cm}$ intervals. Note that the $2.5-\mathrm{cm}$ interval does not limit the range estimation accuracy in the unsaturated case, where interpolation is possible from Fig. 7.

\section{Experimental Verification and Discussion}

In this section, we experimentally verify the proposed method by situating targets at randomly selected distances $r$ and azimuth angles $\theta$ and collecting a total of 194 test scans. The targets are randomly located at azimuth angles varying from -45 to $45 \mathrm{deg}$ from their nearest to their maximum observable ranges in Figs. 4, 5, and 6.

The results of least-squares-based target differentiation are displayed in Tables 1 and 2 in the form of confusion matrices. Table 1 gives the results obtained using the maximum intensity (or the middle-of-two-maxima intensity for corner) values, and Table 2 gives those obtained using the intensity value at the COG of the scans. The average accuracy over all target types can be found by summing the correct decisions given along the diagonal of the confusion matrix and dividing this sum by the total number of test trials (194). The same average correct classification rate is achieved by using the maximum and the COG variations of the least-squares approach, which is $77 \%$.

Matched filter differentiation results are presented in Table 3. The average accuracy of differentiation over all target types is $80 \%$, which is better than that obtained with the least-squares approach.

Planes and corners covered with aluminum are correctly classified with all approaches employed due to their distinctive features. Planar targets of different surface properties are better classified than the others, with a correct differentiation rate of $91 \%$ for the matched filtering approach. For corner targets, the highest correct differentiation rate of $83 \%$ is achieved with the COG variation of the leastsquares approach. The greatest difficulty is encountered in the differentiation of edges of different surfaces, which have the most similar intensity patterns. The highest correct differentiation rate of $60 \%$ for edges is achieved with the maximum intensity variation of the least-squares approach. 
Aytaç and Barshan: Simultaneous extraction of geometry ...

Table 1 Confusion matrix: least-squares-based classification (maximum variation).

\begin{tabular}{lcccccccccccc}
\hline \hline & & \multicolumn{1}{c}{ Detected } \\
\cline { 3 - 11 } Actual & & AL & WC & ST & AL & WC & ST & AL & WC & ST \\
\hline & AL & 24 & - & - & - & - & - & - & - & - \\
P & WC & - & 25 & 4 & - & - & - & - & - & - \\
& ST & - & 9 & 20 & - & - & - & - & - & - \\
& AL & - & - & - & 22 & - & - & - & - & - \\
C & WC & - & - & - & - & 10 & 12 & - & - & - \\
& ST & - & - & - & - & - & 20 & - & - & - \\
& AL & - & - & - & - & - & - & 9 & - & 1 \\
& E & WC & - & - & - & - & - & - & - & 11 & 9 \\
& ST & - & - & 1 & - & - & - & - & 8 & 9
\end{tabular}

$\overline{\mathrm{AL} \text {, aluminum; WC, white cloth; ST, Styrofoam; WO, wood; BR, }}$ brown paper; VI, violet paper; $\mathrm{BL}$, black paper; $\mathrm{WH}$, white paper; $\mathrm{P}$, plane; C, corner; E, edge; CY, cylinder.

Taken separately, the geometry and surface type of targets can be correctly classified with rates of 99 and $81 \%$, respectively, which shows that the geometrical properties of the targets are more distinctive than their surface properties, and surface determination is the limiting factor.

The average absolute range and azimuth estimation errors for the different approaches are presented in Table 4 for all test targets. As we see in the table, using the maximum and COG variations of the least-squares approach, the target ranges are estimated with average absolute range errors of 1.8 and $1.7 \mathrm{~cm}$, respectively. Matched filtering results in an average absolute range error of $1.5 \mathrm{~cm}$, which is better than the least-squares approach. The greatest contribution to the range errors comes from targets which are incorrectly differentiated and/or whose intensity scans are saturated. If we average over only correctly differentiated targets (regardless of whether they lead to saturation), the average absolute range errors are reduced to $1.2,1.0$, and $0.7 \mathrm{~cm}$ for the maximum and COG variations of the leastsquares and the matched filtering approaches, respectively. As for azimuth estimation, the respective average absolute errors for the maximum and COG variations of leastsquares and the matched filtering approaches are 1.6, 1.5, and $1.1 \mathrm{deg}$, with matched filtering resulting in the smallest error. When we average over only correctly differentiated targets, these errors are reduced to 1.5, 1.2, and $0.9 \mathrm{deg}$, respectively.

To explore the boundaries of system performance and to assess the robustness of the system, we also tested the system with targets of either unfamiliar geometry, unfamiliar surface, or both, whose scans are not included in the reference data sets. Therefore, these targets are totally new to the system. First, tests were done for planes, corners, and edges covered with five new surfaces: brown, violet, black, and white paper, and wood. The results of these tests are presented in Tables 5, 6, and 7. Planes are classified as planes $100 \%$ of the time using both variations of the leastsquares method and $99.3 \%$ of the time using the matched filtering approach. Corners are classified as corners 100\% of the time using any of the three approaches. Edges are correctly classified $89.1 \%$ of the time using the maximum
Table 2 Confusion matrix: least-squares based classification (COG variation).

\begin{tabular}{lccccccccccc}
\hline \hline & & \multicolumn{1}{c}{ Detected } \\
\cline { 3 - 11 } Actual & & AL & WC & ST & AL & WC & ST & AL & WC & ST \\
\hline & AL & 24 & - & - & - & - & - & - & - & - \\
P & WC & - & 25 & 4 & - & - & - & - & - & - \\
& ST & - & 9 & 20 & - & - & - & - & - & - \\
& AL & - & - & - & 22 & - & - & - & - & - \\
C & WC & - & - & - & - & 13 & 9 & - & - & - \\
& ST & - & - & - & - & 2 & 18 & - & - & - \\
& AL & - & - & 1 & - & - & - & 7 & - & 2 \\
E & WC & - & - & - & - & - & - & - & 14 & 6 \\
& ST & - & 1 & 1 & - & - & - & - & 10 & 6
\end{tabular}

$\overline{\overline{\text { AL, aluminum; WC, white cloth; ST, Styrofoam; WO, wood; BR, }}}$ brown paper; $\mathrm{VI}$, violet paper; $\mathrm{BL}$, black paper; $\mathrm{WH}$, white paper; $\mathrm{P}$, plane; C, corner; E, edge; CY, cylinder.

variation of the least-squares approach, $88.2 \%$ of the time using the COG variation of the least-squares approach, and $87.3 \%$ of the time using the matched filtering approach. In these tests, no target type is mistakenly classified as a corner due to the unique characteristics of the corner scans. For the same reason, corners of the preceding five surface types are never classified as planes or edges. The range and azimuth errors are comparable or slightly larger than before (not shown).

We also tested the system with cylinders, which were not among the three geometries in the original data sets, with the same surface types as used in the reference data sets: aluminum, white cloth, and Styrofoam. The results are given in Table 8 and indicate that cylindrical targets are most likely to be classified as edges. In this case, correct surface classification rate drops to $35 \%$. We have also considered cylinders whose surface properties are different than the surface types considered in the reference data sets. These are brown, violet, black, and white paper and wood. That is, both the geometry and surface type of this target is

Table 3 Confusion matrix: matched filter based classification.

\begin{tabular}{lccccccccccc}
\hline \hline & & \multicolumn{1}{c}{ Detected } \\
\cline { 3 - 11 } Actual & & AL & WC & ST & AL & WC & ST & AL & WC & ST \\
\hline & AL & 24 & - & - & - & - & - & - & - & - \\
P & WC & - & 27 & 2 & - & - & - & - & - & - \\
& ST & - & 5 & 24 & - & - & - & - & - & - \\
& AL & - & - & - & 22 & - & - & - & - & - \\
C & WC & - & - & - & - & 14 & 8 & - & - & - \\
& ST & - & - & - & - & 4 & 16 & - & - & - \\
& AL & - & - & - & - & - & - & 9 & 1 & - \\
& E & WC & - & - & - & - & - & - & - & 11 & 9 \\
& ST & - & - & 2 & - & - & - & - & 8 & 8 \\
\hline \hline
\end{tabular}

$\overline{\mathrm{AL} \text {, aluminum; WC, white cloth; ST, Styrofoam; WO, wood; BR, }}$ brown paper; VI, violet paper; $\mathrm{BL}$, black paper; $\mathrm{WH}$, white paper; $\mathrm{P}$, plane; C, corner; E, edge; CY, cylinder. 
Aytaç and Barshan: Simultaneous extraction of geometry ...

Table 4 Absolute range and azimuth estimation errors over all test targets.

\begin{tabular}{|c|c|c|c|c|c|c|c|c|c|c|c|}
\hline \multirow[b]{2}{*}{ Method } & & \multicolumn{3}{|c|}{$\mathrm{P}$} & \multicolumn{3}{|c|}{ C } & \multicolumn{3}{|c|}{$E$} & \multirow[b]{2}{*}{ Average Erro } \\
\hline & & $\mathrm{AL}$ & WC & ST & $\mathrm{AL}$ & WC & ST & $\mathrm{AL}$ & WC & ST & \\
\hline \multirow[t]{2}{*}{ LS-max } & $r(\mathrm{~cm})$ & 2.2 & 2.3 & 1.0 & 2.1 & 0.8 & 0.5 & 2.4 & 1.9 & 2.7 & 1.8 \\
\hline & $\theta(\mathrm{deg})$ & 0.9 & 2.3 & 0.8 & 2.4 & 1.7 & 1.3 & 1.1 & 2.0 & 1.7 & 1.6 \\
\hline \multirow[t]{2}{*}{ LS-COG } & $r(\mathrm{~cm})$ & 2.2 & 0.6 & 1.0 & 2.1 & 0.6 & 0.6 & 3.8 & 1.4 & 3.2 & 1.7 \\
\hline & $\theta(\mathrm{deg})$ & 0.9 & 1.0 & 0.8 & 2.4 & 1.4 & 1.1 & 1.2 & 2.2 & 2.3 & 1.5 \\
\hline \multirow[t]{2}{*}{ MF } & $r(\mathrm{~cm})$ & 1.7 & 0.5 & 0.7 & 1.5 & 0.6 & 0.6 & 2.2 & 1.7 & 4.2 & 1.5 \\
\hline & $\theta(\mathrm{deg})$ & 0.8 & 0.9 & 0.7 & 1.0 & 1.1 & 1.0 & 1.1 & 2.6 & 0.9 & 1.1 \\
\hline
\end{tabular}

LS: least-squares, MF: matched filter.

totally unfamiliar to the system. Again, cylinders are most likely to be classified as edges with Styrofoam surface type (see Table 9). In these two cases, average range estimation error increases to about 9 to $11 \mathrm{~cm}$, but the azimuth error is of the same order of magnitude as before, since our azimuth estimation method is independent of target type.

These results indicate that geometrical properties of the targets are more dominant and distinctive compared to their surface properties. When the geometry is familiar but the surface type is not, as in the cases in Tables 5, 6, and 7, the correct classification rate of the geometry is very high (about $96 \%$ on the average). However, when the surface type is familiar but the geometry is not, the correct classification rate of the surface type is lower $(35 \%)$, as in Table 8.

Among the three approaches, the maximum variation of the least-squares approach is slightly more robust to deviations from targets included in the reference sets.

In the remainder of this section, we discuss the effect of varying the orientation of the targets from their head-on

Table 5 Confusion matrix for planar targets with unfamiliar surface.

\begin{tabular}{|c|c|c|c|c|c|c|c|c|c|c|}
\hline \multirow[b]{3}{*}{ Actual } & & \multicolumn{9}{|c|}{ Detected } \\
\hline & & \multicolumn{3}{|c|}{$P$} & \multicolumn{3}{|c|}{$C$} & \multicolumn{3}{|c|}{$E$} \\
\hline & & $A L$ & WC & ST & $\mathrm{AL}$ & WC & ST & $A L$ & WC & ST \\
\hline & WO & - & 16 & 14 & - & - & - & - & - & \\
\hline & $\mathrm{BR}$ & - & 20 & 10 & - & - & - & - & - & - \\
\hline$P$ & VI & - & 22 & 8 & - & - & - & - & - & - \\
\hline \multirow[t]{4}{*}{ (LS-max) } & $\mathrm{BL}$ & - & 24 & 6 & - & - & - & - & - & - \\
\hline & WH & - & 18 & 11 & - & - & - & - & - & - \\
\hline & WO & - & 15 & 15 & - & - & - & - & - & - \\
\hline & $\mathrm{BR}$ & - & 20 & 10 & - & - & - & - & - & - \\
\hline$P$ & VI & - & 22 & 8 & - & - & - & - & - & - \\
\hline \multirow[t]{4}{*}{ (LS-COG) } & $B L$ & - & 24 & 6 & - & - & - & - & - & - \\
\hline & $\mathrm{WH}$ & - & 16 & 13 & - & - & - & - & - & - \\
\hline & WO & - & 19 & 11 & - & - & - & - & - & - \\
\hline & $\mathrm{BR}$ & - & 22 & 8 & - & - & - & - & - & - \\
\hline$P$ & VI & - & 23 & 6 & - & - & - & - & - & 1 \\
\hline \multirow[t]{2}{*}{ (MF) } & $\mathrm{BL}$ & 1 & 25 & 4 & - & - & - & - & - & - \\
\hline & WH & - & 18 & 11 & - & - & - & - & - & - \\
\hline
\end{tabular}

$\overline{\mathrm{AL} \text {, aluminum; WC, white cloth; ST, Styrofoam; WO, wood; BR, }}$ brown paper; VI, violet paper; $\mathrm{BL}$, black paper; $\mathrm{WH}$, white paper; $\mathrm{P}$, plane; C, corner; E, edge; CY, cylinder. positions. This constitutes a separate degree of freedom than the range and azimuth of the targets. Varying the orientation for planes does not make any difference since a complete scan is acquired. The acquired scan will still be that of a plane, with its peak shifted to the azimuthal value, which corresponds to the direction where the sensor line of sight is perpendicular to the plane. In other words, varying the orientation of planes does not lead to any deterioration in performance since such planes are already included in the reference set. Variation of orientation is not an issue for cylinders to begin with, since they are rotation invariant.

Change of orientation will make a difference when the target geometry is a corner or an edge, leading to scans not existing in the reference set. Unlike with the case of planes and cylinders, varying the orientation of corners and edges leads to asymmetric scans. If the scan is symmetric, it is either a plane or a cylinder, or a corner or an edge with nearly 0 deg orientation, and the described algorithm can handle it. If the scan is asymmetric, we know that the target is either a corner or an edge with nonzero orientation.

Table 6 Confusion matrix for corner targets with unfamiliar surface.

\begin{tabular}{|c|c|c|c|c|c|c|c|c|c|c|}
\hline \multirow[b]{3}{*}{ Actual } & & \multicolumn{9}{|c|}{ Detected } \\
\hline & & \multicolumn{3}{|c|}{$P$} & \multicolumn{3}{|c|}{$\mathrm{C}$} & \multicolumn{3}{|c|}{ E } \\
\hline & & $\mathrm{AL}$ & WC & ST & $\mathrm{AL}$ & WC & ST & $\mathrm{AL}$ & WC & ST \\
\hline & WO & - & - & - & - & 9 & 13 & - & - & - \\
\hline & $\mathrm{BR}$ & - & - & - & 1 & 3 & 17 & - & - & - \\
\hline C & VI & - & - & - & 1 & 20 & - & - & - & - \\
\hline \multirow{4}{*}{ (LS-max) } & $\mathrm{BL}$ & - & - & - & - & 12 & 10 & - & - & - \\
\hline & WH & - & - & - & - & 12 & 9 & - & - & - \\
\hline & wo & - & - & - & - & 10 & 12 & - & - & - \\
\hline & $\mathrm{BR}$ & - & - & - & 1 & 3 & 17 & - & - & - \\
\hline $\mathrm{C}$ & VI & - & - & - & 1 & 2 & 18 & - & - & - \\
\hline \multirow[t]{4}{*}{ (LS-COG) } & $\mathrm{BL}$ & - & - & - & - & 13 & 9 & - & - & - \\
\hline & $\mathrm{WH}$ & - & - & - & - & 13 & 8 & - & - & - \\
\hline & wo & - & - & - & - & 14 & 8 & - & - & - \\
\hline & $\mathrm{BR}$ & - & - & - & 1 & 4 & 16 & - & - & - \\
\hline $\mathrm{C}$ & VI & - & - & - & 1 & 3 & 17 & - & - & - \\
\hline \multirow[t]{2}{*}{ (MF) } & $\mathrm{BL}$ & - & - & - & - & 13 & 9 & - & - & - \\
\hline & WH & - & - & - & - & 13 & 8 & - & - & - \\
\hline
\end{tabular}

$\overline{\mathrm{AL} \text {, aluminum; WC, white cloth; ST, Styrofoam; WO, wood; BR, }}$ brown paper; VI, violet paper; $\mathrm{BL}$, black paper; $\mathrm{WH}$, white paper; $\mathrm{P}$, plane; C, corner; E, edge; CY, cylinder. 
Aytaç and Barshan: Simultaneous extraction of geometry ...

Table 7 Confusion matrix for edge targets with unfamiliar surface.

\begin{tabular}{|c|c|c|c|c|c|c|c|c|c|c|}
\hline \multirow[b]{3}{*}{ Actual } & & \multicolumn{9}{|c|}{ Detected } \\
\hline & & \multicolumn{3}{|c|}{$\mathrm{P}$} & \multicolumn{3}{|c|}{ C } & \multicolumn{3}{|c|}{$E$} \\
\hline & & $\mathrm{AL}$ & WC & ST & $A L$ & WC & ST & $\mathrm{AL}$ & WC & ST \\
\hline & WO & - & 1 & 5 & - & - & - & - & 9 & 7 \\
\hline & $\mathrm{BR}$ & - & - & 2 & - & - & - & - & 12 & 8 \\
\hline E & VI & - & - & 2 & - & - & - & - & 10 & 8 \\
\hline \multirow[t]{4}{*}{ (LS-max) } & $\mathrm{BL}$ & - & - & - & - & - & - & - & 14 & 9 \\
\hline & WH & - & - & 2 & - & - & - & - & 12 & 9 \\
\hline & WO & - & 2 & 4 & - & - & - & 1 & 11 & 4 \\
\hline & $\mathrm{BR}$ & - & - & - & - & - & - & 1 & 15 & 6 \\
\hline$E$ & VI & - & 1 & 3 & - & - & - & - & 15 & 1 \\
\hline \multirow[t]{4}{*}{ (LS-COG) } & $\mathrm{BL}$ & - & 1 & - & - & - & - & - & 16 & 6 \\
\hline & $\mathrm{WH}$ & - & 2 & - & - & - & - & - & 13 & 8 \\
\hline & WO & - & - & 6 & - & - & - & - & 12 & 4 \\
\hline & $\mathrm{BR}$ & - & - & 3 & - & - & - & - & 10 & 9 \\
\hline E & VI & - & - & 1 & - & - & - & - & 17 & 2 \\
\hline \multirow[t]{2}{*}{ (MF) } & $\mathrm{BL}$ & - & - & 2 & - & - & - & - & 15 & 6 \\
\hline & $\mathrm{WH}$ & - & - & 2 & - & - & - & - & 12 & 9 \\
\hline
\end{tabular}

AL, aluminum; WC, white cloth; ST, Styrofoam; WO, wood; BR, brown paper; $\mathrm{VI}$, violet paper; $\mathrm{BL}$, black paper; $\mathrm{WH}$, white paper; $\mathrm{P}$, plane; C, corner; E, edge; CY, cylinder.

While it is possible to deal with this case by extending the reference set to include targets with nonzero orientation, the introduction of a simple rule enables us to handle such cases with only minor modification of the already presented algorithm. We can determine whether the asymmetric scan comes from a corner or an edge by checking whether or not it has two humps. Thus, even with arbitrary orientations, the target geometry can be determined. Furthermore, we observe that variations in orientation have very little effect on the central intensity of the asymmetric scans (see Fig. 8 for some examples). This means that the central intensity value can be used to determine the distance in the same

Table 8 Confusion matrix for cylindrical targets with familiar surface.

\begin{tabular}{lccccccccccc}
\hline \hline & & \multicolumn{1}{c}{ Detected } \\
\cline { 3 - 10 } Actual & & AL & WC & ST & AL & WC & ST & AL & WC & ST \\
\hline & AL & - & - & - & - & - & - & 1 & - & 12 \\
CY & WC & 7 & - & 1 & - & - & - & - & 5 & 12 \\
(LS-max) & ST & 4 & - & - & - & - & - & 1 & 4 & 16 \\
& AL & - & - & - & - & - & - & - & - & 13 \\
CY & WC & 7 & 1 & - & - & - & - & - & 4 & 13 \\
(LS-COG) & ST & 4 & 1 & 1 & - & - & - & - & 5 & 14 \\
& AL & - & - & - & - & - & - & 1 & - & 12 \\
CY & WC & 8 & - & 2 & - & - & - & - & 2 & 13 \\
(MF) & ST & 5 & - & 1 & - & - & - & - & 5 & 14 \\
\hline \hline AL, & & & & & & & & & & &
\end{tabular}

AL, aluminum; WC, white cloth; ST, Styrofoam; WO, wood; BR, brown paper; VI, violet paper; $\mathrm{BL}$, black paper; $\mathrm{WH}$, white paper; $\mathrm{P}$, plane; C, corner; E, edge; CY, cylinder.
Table 9 Confusion matrix for cylindrical targets with unfamiliar surface.

\begin{tabular}{|c|c|c|c|c|c|c|c|c|c|c|}
\hline \multirow[b]{3}{*}{ Actual } & & \multicolumn{9}{|c|}{ Detected } \\
\hline & & \multicolumn{3}{|c|}{$P$} & \multicolumn{3}{|c|}{ C } & \multicolumn{3}{|c|}{$E$} \\
\hline & & $A L$ & WC & ST & $A L$ & WC & ST & $A L$ & WC & ST \\
\hline & WO & 8 & - & - & - & - & - & - & 4 & 13 \\
\hline & $\mathrm{BR}$ & 7 & - & - & - & - & - & - & 5 & 13 \\
\hline CY & VI & 7 & 1 & 1 & - & - & - & - & 5 & 12 \\
\hline \multirow[t]{4}{*}{ (LS-max) } & $\mathrm{BL}$ & 5 & - & - & - & - & - & - & 3 & 16 \\
\hline & $\mathrm{WH}$ & 8 & - & - & - & - & - & - & 5 & 13 \\
\hline & WO & 8 & - & - & - & - & - & - & 3 & 14 \\
\hline & $\mathrm{BR}$ & 7 & - & 1 & - & - & - & - & 4 & 13 \\
\hline CY & VI & 7 & 2 & 1 & - & - & - & - & 5 & 11 \\
\hline \multirow[t]{4}{*}{ (LS-COG) } & $\mathrm{BL}$ & 5 & - & - & - & - & - & 1 & 7 & 11 \\
\hline & $\mathrm{WH}$ & 8 & 1 & - & - & - & - & - & 3 & 14 \\
\hline & WO & 8 & - & - & - & - & - & - & 5 & 12 \\
\hline & $\mathrm{BR}$ & 7 & - & 2 & - & - & - & - & 4 & 12 \\
\hline CY & VI & 8 & - & 3 & - & - & - & - & 3 & 12 \\
\hline \multirow[t]{2}{*}{ (MF) } & $\mathrm{BL}$ & 7 & - & 2 & - & - & - & - & 3 & 12 \\
\hline & $\mathrm{WH}$ & 8 & - & - & - & - & - & - & 5 & 13 \\
\hline
\end{tabular}

AL, aluminum; WC, white cloth; ST, Styrofoam; WO, wood; BR, brown paper; $\mathrm{VI}$, violet paper; $\mathrm{BL}$, black paper; $\mathrm{WH}$, white paper; $\mathrm{P}$, plane; C, corner; E, edge; CY, cylinder.

manner as before by using linear interpolation on the central intensity versus distance curves for a particular target.

To summarize, with the preceding observations and minor modifications to the algorithm, the same geometry and surface recognition and position estimation objectives can be achieved even when the targets do not have 0-deg orientations. Note, however, that while this approach enables us to accomplish the desired objectives in an orientationinvariant manner, it does not determine the orientation of the target. If determination of target orientation is also desired, this can be accomplished either by storing corresponding scans in the reference set (increasing storage requirements), or more efficiently by constructing orientation angle versus measure-of-asymmetry plots based on suitable measures of asymmetry (for instance, ratios of characteristics of the left- and right-hand sides of the scans).

To demonstrate this, we performed additional experiments with corners and edges. These targets were placed at random orientation angles at randomly selected distances. A total of 100 test scans were collected. Using the orientationinvariant approach already described, $100 \%$ correct differentiation and absolute mean range errors of 1.02 and 1.47 $\mathrm{cm}$ for corners and edges respectively, were achieved.

We also tested the case where reference scans corresponding to different orientations are acquired. Reference data sets were collected for both targets with 5 -cm distance increments at $\theta=0 \mathrm{deg}$, where the orientation of the targets are varied between -35 to 35 deg with 2.5-deg increments. A total of 489 reference scans were collected. For each test scan, the best-fitting reference scan was found by matched filtering. This method also resulted in 100\% correct differentiation rate. Absolute mean range and orientation errors 


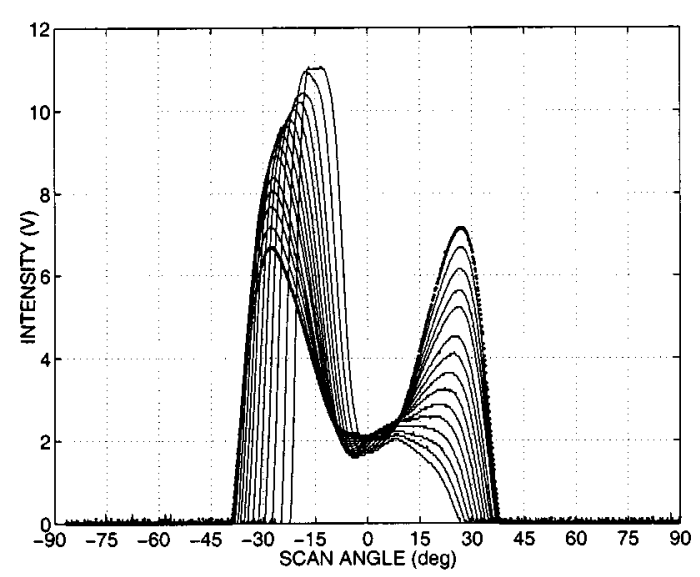

(a)

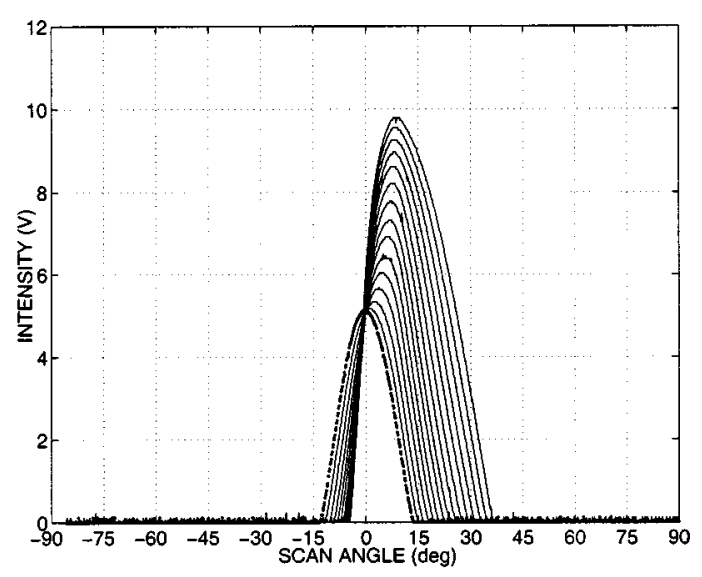

(b)

Fig. 8 Intensity scans for a wooden (a) corner at $65 \mathrm{~cm}$ and (b) edge at $35 \mathrm{~cm}$ for orientations between 0 and 35 deg with 2.5-deg increments. The curves with the dotted lines indicate 0 -deg orientation.

for corners and edges were 1.13 and $1.26 \mathrm{~cm}$ and 4.48 and $5.53 \mathrm{deg}$, respectively.

\section{Discussion and Conclusion}

In this study, differentiation and localization of commonly encountered indoor features or targets such as planes, corners, and edges with different surfaces was achieved using an inexpensive IR emitter and detector pair. Different approaches were compared in terms of correct target differentiation rate, and range and azimuth estimation accuracy. The matched filtering approach in general gave better results for both differentiation and localization. The robustness of the methods was investigated by presenting the system with targets of either unfamiliar geometry, unfamiliar surface type, or both. These targets were not included in the reference sets so they were completely new to the system.

The accomplishment of this study is that even though the intensity scan patterns are highly dependent on target location, and this dependence cannot be represented by a simple relationship, we realize position-invariant target differentiation. An average correct target differentiation rate of $80 \%$ over all target types was achieved and targets were localized within absolute range and azimuth errors of 1.5 $\mathrm{cm}$ and $1.1 \mathrm{deg}$, respectively. The method we propose is scalable in the sense that the accuracy can be increased by increasing the number of reference scans without increasing the computational cost. The results reported here represent the outcome of our efforts to explore the limits of what is achievable in terms of identifying information with only a simple emitter-detector pair. Such simple sensors are usually put to much lower information-extracting uses.

We saw that the geometrical properties of the targets are more distinctive than their surface properties, and surface determination is the limiting factor. In this paper, we demonstrated target differentiation for three target geometries and three different surfaces. Based on the data we collected and on our previous works, ${ }^{34-36}$ it seems possible to increase the vocabulary of different geometries, provided they are not too similar. However, the same cannot be said for the number of different surfaces. For a given total num- ber of distinct targets, increasing the number of surfaces and decreasing the number of geometries will, in general, worsen the results. On the other hand, decreasing the number of surfaces and increasing the number of geometries will, in general, improve the results.

This paper demonstrated that simple IR sensors, when coupled with appropriate processing, can be used to extract substantially more information than such devices are commonly employed for. We expect this flexibility to significantly extend the range of applications in which such lowcost single-sensor-based systems can be used. Specifically, we expect that it will be possible to go beyond relatively simple tasks such as simple object and proximity detection, counting, distance and depth monitoring, floor sensing, position measurement, obstacle/collision avoidance, and dealing with tasks such as differentiation, classification, recognition, clustering, position estimation, map building, perception of the environment and surroundings, autonomous navigation, and target tracking. The approach presented here would be more useful where a self-correcting operation is possible due to repeated observations and feedback.

A typical application of the demonstrated system would be in mobile robotics in surveying an unknown environment composed of elementary features or targets. Many artificial environments fall into this category. Industrial applications where different targets and/or materials must be identified and separated may also benefit from this approach. We plan to test and evaluate the developed system on a small mobile robot in our laboratory for map building in a test room composed of the primitive features considered in this study.

Current work involves identifying more generally shaped targets (such as a vase or a bottle) by using several scans from each target obtained at different heights. Also being considered is the parametric modeling and representation of intensity scans rather than the use of the intensity scan vectors themselves. 


\section{Acknowledgments}

This research was supported by TÜBITAK under BDP and 197E051 grants. The authors would like to thank the Department of Engineering Science of the University of Oxford for donating the IR sensors.

\section{References}

1. P. J. Phillips, "Matching pursuit filters applied to face identification," IEEE Trans. Image Process. 7, 1150-1164 (Aug. 1998).

2. H. Kwon, S. Z. Der, and N. M. Nasrabadi, "Adaptive multisensor target detection using feature-based fusion," Opt. Eng. 41, 69-80 (Jan. 2002)

3. T. Tsao and Z. Q. Wen, "Image-based target tracking through rapid sensor orientation change," Opt. Eng. 41, 697-703 (Mar. 2002).

4. I. Pavlidis, P. Symosek, B. Fritz, M. Bazakos, and N. Papanikolopoulos, "Automatic detection of vehicle occupants: the imaging problem and its solution," Mach. Vision Appl. 11, 313-320 (Apr. 2000).

5. P. M. Tag, R. L. Bankert, and L. R. Brody, "An AVHRR multiple cloud-type classification package," J. Appl. Meterol. 39, 125-134 (Feb. 2000)

6. A. K. Jain, N. K. Ratha, and S. Lakshmanan, "Object detection using Gabor filters," Pattern Recogn. 30, 295-309 (Feb. 1997).

7. Z. Zalevsky, D. Mendlovic, E. Rivlin, and S. Rotman, "Contrasted statistical processing algorithm for obtaining improved target detection performances in infrared cluttered environment," Opt. Eng. 39, 2609-2617 (Oct. 2000).

8. B. Bhanu, P. Symosek, and S. Das, "Analysis of terrain using multispectral images," Pattern Recogn. 30, 197-215 (Feb. 1997).

9. F. T. S. Yu and S. Yin, Eds., Selected Papers on Optical Pattern Recognition, Vol. MS 156 of SPIE Milestone Series, SPIE Optical Engineering Press, Bellingham, WA (1999).

10. D. Casasent and D. Psaltis, "Scale invariant optical correlation using Mellin transforms," Opt. Commun. 17, 59-63 (Apr. 1976).

11. M. McDonnell, "Clarification on use of Mellin transform in optical pattern recognition," Opt. Commun. 25(3), 320-322 (1978).

12. H. H. Arsenault, Y. N. Hsu, and K. Chalasinska-Macukow, "Rotationinvariant pattern recognition," Opt. Eng. 23, 705-709 (Nov./Dec. 1984).

13. F. T. S. Yu, X. Li, E. Tam, S. Jutamulia, and D. A. Gregory, "Rotation invariant pattern recognition with a programmable joint transform correlator," Appl. Opt. 28, 4725-4727 (Nov. 1989).

14. G. Gheen, "Design considerations for low-clutter, distortion invariant correlation filters," Opt. Eng. 29, 1029-1032 (Sep. 1990).

15. C. Gu, J. Hong, and S. Campbell, "2-D shift invariant volume holographic correlator," Opt. Commun. 88, 309-314 (Apr. 1992).

16. P. Refregier, "Optical pattern recognition—optimal trade-off circular harmonic filters," Opt. Commun. 86, 113-118 (Nov. 1991).

17. E. Cheung and V. J. Lumelsky, "Proximity sensing in robot manipulator motion planning: system and implementation issues," IEEE Trans. Rob. Autom. 5, 740-751 (Dec. 1989).

18. A. J. Hand, "Infrared sensor counts insects," Photonics Spectra 32, 30-31 (Nov. 1998)

19. H. C. Wikle, S. Kottilingam, R. H. Zee, and B. A. Chin, "Infrared sensing techniques for penetration depth control of the submerged arc welding process," J. Mater. Process. Technol. 113, 228-233 (June 2001).

20. Y. Arai and M. Sekiai, "Absolute position measurement system for mobile robot based on incident angle detection of infrared light," in Proc. of the IEEE/RSJ Int. Conf. on Intelligent Robots and Systems, pp. 986-991, Las Vegas, NV (2003).

21. B. Butkiewicz, "Position control system with fuzzy microprocessor AL220," Lect. Notes Comput. Sci. 1226, 74-81 (1997).

22. V. J. Lumelsky and E. Cheung, "Real-time collision avoidance in teleoperated whole-sensitive robot arm manipulators," IEEE Trans. Syst. Man. Cybern. 23, 194-203 (Jan./Feb. 1993).

23. T.-H. S. Li, S.-J. Chang, and Y.-X. Chen, "Implementation of autonomous fuzzy garage-parking control by an FPGA-based car-like mobile robot using infrared sensors," in Proc. IEEE Int. Conf. on Robotics and Automation, pp. 3776-3781, Taipei, Taiwan (2003).

24. H.-H. Kim, Y.-S. Ha, and G.-G. Jin, "A study on the environmental map building for a mobile robot using infrared range-finder sensors," in Proc. IEEE/RSJ Int. Conf. on Intelligent Robots and Systems, pp. 711-716, Las Vegas, NV (2003).

25. A. M. Flynn, "Combining sonar and infrared sensors for mobile robot navigation," Int. J. Robot. Res. 7, 5-14 (Dec. 1988).

26. P. M. Novotny and N. J. Ferrier, "Using infrared sensors and the
Phong illumination model to measure distances," in Proc. IEEE Int Conf. on Robotics and Automation, pp. 1644-1649, Detroit, MI (1999).

27. G. Benet, F. Blanes, J. E. Simó, and P. Pérez, "Using infrared sensors for distance measurement in mobile robots," Robt. Auton. Syst. 40, 255-266 (2002).

28. F. J. Cuevas, M. Servin, and R. Rodriguez-Vera, "Depth object recovery using radial basis functions," Opt. Commun. 163, 270-277 (May 1999).

29. P. Klysubun, G. Indebetouw, T. Kim, and T. C. Poon, "Accuracy of three-dimensional remote target location using scanning holographic correlation," Opt. Commun. 184, 357-366 (Oct. 2000).

30. J. J. Esteve-Taboada, P. Refregier, J. Garcia, and C. Ferreira, "Targe localization in the three-dimensional space by wavelength mixing," Opt. Commun. 202, 69-79 (Feb. 2002).

31. B. Iske, B. Jäger, and U. Rückert, "A ray-tracing approach for simulating recognition abilities of active infrared sensor arrays," in Proc. 1st IEEE Int. Conf. on Sensors, Vol. 2, pp. 1227-1232, Orlando, FL (2002).

32. K. Hashimoto, T. Tsuruta, K. Morinaka, and N. Yoshiike, "High performance human information sensor," Sens. Actuators, A 79, 46-52 (Jan. 2000).

33. D. M. Scott, "A 2-color near-infrared sensor for sorting recycled plastic waste," Meas. Sci. Technol. 6, 156-159 (Feb. 1995).

34. T. Aytaç and B. Barshan, "Differentiation and localization of targets using infrared sensors," Opt. Commun. 210, 25-35 (Sep. 2002).

35. T. Aytaç and B. Barshan, "Rule-based target differentiation and position estimation based on infrared intensity measurements," Opt. Eng. 42, 1766-1771 (June 2003)

36. B. Barshan and T. Aytaç, "Position-invariant surface recognition and localization using infrared sensors," Opt. Eng. 42, 3589-3594 (Dec. 2003).

37. Matrix Elektronik, AG, Kirchweg 24 CH-5422 Oberehrendingen, Switzerland, IRS-U-4A Proximity Switch Datasheet (1995).

38. Arrick Robotics, P.O. Box 1574, Hurst, Texas, 76053 URL www.robotics.com/rt12.html, RT-12 Rotary Positioning Table (2002)

39. J. W. Goodman, Introduction to Fourier Optics, 2nd ed., pp. 246-249, McGraw-Hill, New York (1996)

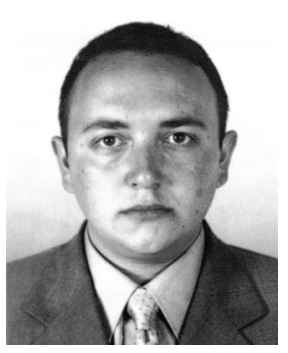

Tayfun Aytaç received his BS degree in electrical engineering in 2000 from Gaz University, Ankara, Turkey, and his MS degree in electrical engineering in 2002 from Bilkent University, Ankara, Turkey, where he is currently working toward his $\mathrm{PhD}$ degree. His current research interests include intelligent sensing, optical sensing, pattern recognition, sensor data fusion, target differentiation, and sensor-based robotics.

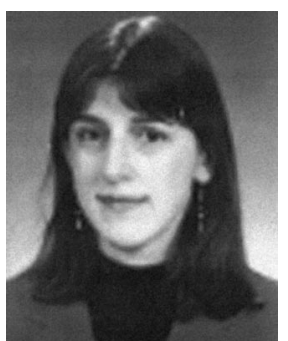

Billur Barshan received her BS degrees in both electrical engineering and physics from Boğaziçi University, Istanbul, Turkey, and her MS and $\mathrm{PhD}$ degrees in electrical engineering from Yale University, New Haven, Connecticut, in 1986, 1988, and 1991 respectively. Dr. Barshan was a research assistant at Yale University from 1987 to 1991, and a postdoctoral researcher with the Robotics Research Group at University of Oxford, United Kingdom, from 1991 to 1993. In 1993, she joined Bilkent University, Ankara, where she is currently a professor in the Department of Electrical Engineering and where she is the founder of the Robotics and Sensing Laboratory. She is the recipient of the 1994 Nakamura Prize awarded to the most outstanding paper at the 1993 IEEE/RSJ Intelligent Robots and Systems International Conference, the 1998 TÜBITAK Young Investigator Award, and the 1999 Mustafa N. Parlar Foundation Research Award. Dr. Barshan's current research interests include in telligent sensors, sonar and inertial navigation systems, sensorbased robotics, and multisensor data fusion. 